\title{
PRESENTACIÓN
}

\section{Infecciones nosocomiales}

L as infecciones nosocomiales son un problema relevante de salud pública de gran trascendencia económica y social, además de constituir un desafío para las instituciones de salud y el personal médico responsable de su atención en las unidades donde se llegan a presentar. Son de importancia clínica y epidemiológica debido a que condicionan altas tasas de morbilidad y mortalidad, e inciden en los años de vida potencialmente perdidos de la población que afectan, a lo cual se suma el incremento en los días de hospitalización y los costos de atención.

Dado que las infecciones nosocomiales son complicaciones en las que se conjugan diversos factores de riesgo que en su mayoría pueden ser susceptibles de prevención y control, las instituciones de salud deben establecer mecanismos eficientes de intervención que permitan la aplicación de medidas preventivas y correctivas encaminadas a la disminución de los factores de riesgo que inciden en la distribución y la frecuencia de dichas infecciones.

Ante ello, se reconoce la necesidad de una mayor participación de los profesionales de las áreas clínica, epidemiológica, de enfermería, laboratorio y de otras especialidades, lo que permitiría, desde una perspectiva multidisciplinaria y de amplio consenso, el estudio integral de las infecciones nosocomiales y su situación actual en México; ello necesariamente incluye la búsqueda y la aplicación de nuevas metodologías y procedimientos operativos que permitan librar con éxito la lucha contra estos padecimientos. En este contexto, la vigilancia epidemiológica y la difusión de sus resultados de información, se inscriben también dentro de estos propósitos, al permitir la aplicación de normas, procedimientos y criterios de trabajo operativo para la identificación temprana y el estudio de las infecciones nosocomiales, así como de otras enfermedades de interés hospitalario.
La vigilancia epidemiológica es un instrumento de apoyo que garantiza el buen funcionamiento de los servicios y coadyuva en la calidad de la atención médica que se otorga a usuarios de todo tipo de servicios. Con este motivo, en México se ha dado inicio a la operación de la Red Hospitalaria de Vigilancia Epidemiológica (RHOVE), como parte de las estrategias nacionales para fortalecer la práctica médica y los procesos de la vigilancia epidemiológica en los hospitales del sector. Actualmente, la RHOVE cuenta con el apoyo de instituciones privadas y organismos internacionales afines, lo que ha permitido su instrumentación y puesta en marcha en el ámbito nacional $y$, por primera vez, se ha podido generar información clínico-epidemiológica útil sobre el patrón de las infecciones nosocomiales y sus factores de riesgo en México. La RHOVE es un esfuerzo institucional de alcance nacional, y es el primero en América Latina que permite la sistematización de la información y el uso de los productos de vigilancia en la solución de los problemas de las unidades locales donde se detectan. La sistematización de las experiencias particulares para la prevención y el control de las infecciones nosocomiales, así como el uso de los productos de información de la vigilancia epidemiológica en la toma de decisiones, es responsabilidad de todo el personal de salud, lo que conlleva su difusión a todos los niveles. Con ello también es posible aportar nuevos conocimientos que se suman a esta iniciativa de difusión de temas relacionados con las infecciones nosocomiales.

De ahí la importancia que conlleva la presente publicación, al integrar, de manera resumida, las experiencias y los conocimientos de profesionales dedicados al estudio, la prevención y el control de las infecciones nosocomiales en México. Con ello, se cumple uno de los objetivos de la práctica médica institucional y la investigación científica, al contemplar el intercambio 
de experiencias y la difusión de los conocimientos, para contender con los problemas de salud y las necesidades de operación asociadas a este tipo de eventos.

En este número especial se presentan temas de relevancia, que resumen los hallazgos de estudios epidemiológicos representativos de la práctica hospitalaria, particularmente en el campo de la prevención y el control, donde las acciones de vigilancia epidemio- lógica e investigación aplicada a las infecciones nosocomiales cobran un alto valor. Con seguridad, los conocimientos que se vierten a partir de los trabajos que aquí se presentan serán de gran utilidad para quienes en el nivel operativo se encuentran a cargo de los programas de vigilancia, prevención y control.

Roberto Tapia Conyer*

\footnotetext{
* Subsecretario de Prevención y Control de Enfermedades, Secretaría de Salud, México.
} 